\title{
Equipo para la determinación de tiempos de almacenamiento de asfalto modificado a condiciones críticas
}

\section{Equipment to determine storage times of modified asphalts at critical conditions}

\section{María Fernanda Abarca Jiménez}

Asistente de Investigación

Unidad de Investigación en Infraestructura y Transporte

LANAMME, Universidad de Costa Rica, San José, Costa Rica

fernanda.abarca@ucr.ac.cr

\section{Rafael Ernesto Villegas Villegas}

Investigador

Unidad de Investigación en Infraestructura y Transporte

LANAMME, Universidad de Costa Rica, San José, Costa Rica

rafael.villegas@ucr.ac.cr

Fecha de recepción: 15 de enero de 2021 / Fecha de aprobación: 06 de mayo de 2021

\section{RESUMEN}

Teniendo el objetivo de estudiar el comportamiento del asfalto modificado durante su almacenamiento, se diseña un tanque para tal propósito dimensionado para su uso en laboratorio. El diseño del tanque pretende mantener la escala dimensional similar a las presentes en los tanques utilizados en la industria para este fin y, a la vez, propiciar la separación de sus fases para analizar el almacenamiento bajo condiciones críticas. Por este motivo, se realiza una comparativa de tanques disponibles comercialmente y sus relaciones dimensionales, además de un estudio sobre las teorías de la sedimentación y separaciones de fase. El principal objetivo de este documento es el diseño mecánico de un tanque de almacenamiento elaborable con materiales de fácil obtención, que permite la toma de muestras necesarias para estudiar el almacenamiento del asfalto modificado a condiciones críticas. Paralelamente, se establecen las bases para el planteamiento de un modelo que aporte en la determinación de tiempos de almacenamiento máximo con la pretensión de verificar la calidad del diseño del asfalto modificado.

Palabras clave: Almacenamiento, asfalto modificado, condiciones críticas de almacenamiento, diseño mecánico de tanques de almacenamiento, separación de fases, tiempo de almacenamiento.

\section{ABSTRACT}

This paper attempts to design a storage tank sized for laboratory use, aiming to study the stability of modified asphalt during storage. The tank design seeks to maintain similar dimensions to those of industrial tanks for asphalt storage. Also, it aims to promote phase separation to analyze the storage under critical conditions. For this reason, this research carries out a comparison of commercially available tanks and their dimensional ratios, as well as studying the theories of sedimentation and phase separation. The main objective of this document is to provide a mechanical design of a storage tank that can be manufactured with accessible materials, which allow to perform the necessary sampling to examine the stability of modified asphalt under critical conditions of storage. In addition, the document establishes bases for the approach of a model that contributes to the determination of maximum storage time. It pretends to verify the quality of the modified asphalt design.

Keywords: Critical storage conditions, mechanical design of storage tanks, modified asphalt, phase separation, storage, storage time. 


\section{INTRODUCCIÓN}

En proyectos que implican la utilización de asfalto modificado cada detalle importa para obtener resultados duraderos que se adecúen a las condiciones de trabajo. Uno de los procesos intermedios al cual se debe prestar mucha atención es al almacenaje. Se sabe que, durante el tiempo de almacenamiento prolongado a altas temperaturas, las mezclas de asfalto con polímeros tienden a separarse en fases (Fu et al., 2006).

Dicha tendencia hace que el tiempo y las condiciones a las cuales se almacena sean sumamente importantes de considerar, ya que las mismas son clave para asegurar las propiedades buscadas en el asfalto modificado. Sin embargo, conocer el comportamiento exacto de la mezcla en el tiempo es complicado y depende de varios factores.

El asfalto puede presentar un comportamiento viscoso, elástico o ambos según la temperatura. Lo anterior, considerando que no se trata solo de asfalto, sino que también entra en juego la presencia de un polímero, puede provocar en los ligantes modificados fenómenos de sedimentación o separación de fases debido a la diferencia de densidades. En consecuencia, resulta en el tanque un enriquecimiento en el ligante de polímeros en la parte inferior (si sedimentan) o en la superior (si floculan), según sus respectivas densidades (Angulo \& Duarte, 2005). Según los mismos autores, Angulo y Duarte (2005), esto puede verse propiciado por una dispersión incorrecta del polímero o por incompatibilidad.

Se ha cuestionado sobre la reversibilidad del proceso de separación del asfalto durante el almacenamiento y se ha concluido que cada parámetro que afecta este fenómeno influye de manera diferente. En el estudio de Rassamdana (Rassamdana, Dabir, Nematy, Farhani, y Sahimi, 1996) se plantea el ejemplo de que, si la división es causada por un aumento en la presión, se puede considerar que el proceso es reversible. De manera contraria, si la separación se debe a efectos de la temperatura o la composición del crudo y el disolvente (en caso de precipitación específicamente), la influencia de estos es más fuerte, ocasionando que la separación sea, al menos, parcialmente reversible (Rassamdana, et al., 1996).

Además, en otras investigaciones como la de Youtcheff y colaboradores, se ha observado la agitación como una práctica beneficiosa para evitar la separación de los componentes del modificado, sin embargo, puede implicar consecuencias como la degradación del asfalto modificado si es utilizada por mucho tiempo (Federal Highway Administration, 2005).
Todo lo anterior resulta de relevancia ya que una separación en la mezcla asfalto-polímero que resulte irreversible o parcialmente irreversible, implica que la calidad del asfalto se verá comprometida, y que pueda ocurrir una degradación en los componentes del material. Entonces, resulta importante considerar la influencia de los factores citados anteriormente durante el almacenamiento.

\section{Tanques de Almacenamiento}

Lo típico para almacenar asfalto modificado es la utilización de depósitos capaces de contener la mezcla en condiciones adecuadas para su posterior venta y/o uso. Estos depósitos o tanques de almacenamiento se fabrican en diferentes diseños, dimensiones y con distintas características según el material que van a contener. Usualmente incorporan dispositivos de agitación o calentamiento para mantener las propiedades del producto por más tiempo.

Generalmente, los tanques de almacenamiento se clasifican en cilíndricos horizontales (Figura 1) y cilíndricos verticales de fondo plano (Figura 2). Carreño y Hernández describen que los primeros son usualmente utilizados para contenidos de volúmenes bajos "debido a que presentan problemas por fallas de corte y flexión" (Carreño \& Hernández, 2008, p. 10). En contraste, los segundos permiten almacenar cantidades más grandes a un bajo costo, con la limitante de que deben usarse a presiones atmosféricas o presiones internas relativamente pequeñas (Carreño \& Hernández, 2008).

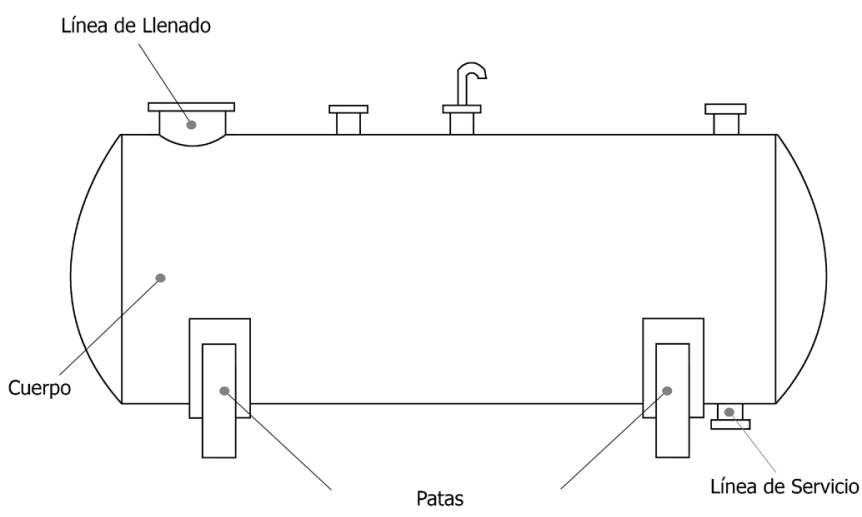

Figura 1. Tanque de almacenamiento horizontal con sus principales componentes señalados 


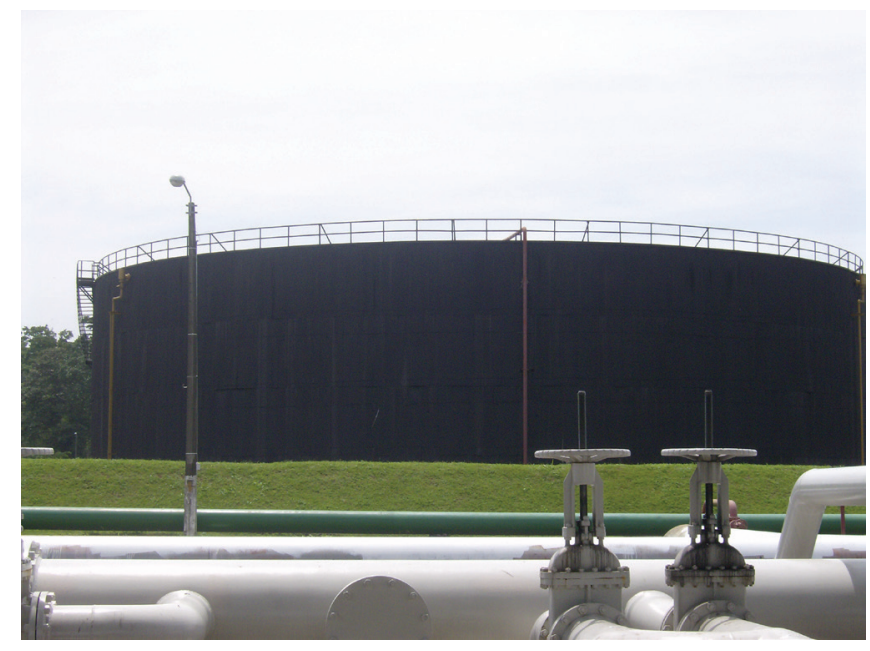

Figura 2. Tanque vertical de fondo plano (Foto cortesía de Daniel Murillo García)

El código API-650 es la base para el diseño, fabricación, montaje, pruebas y operación de los tanques de almacenamiento utilizados en la industria petrolera. Este código detalla las consideraciones y aspectos a cumplir por diseñadores, fabricantes, montadores y operadores (Carreño \& Hernández, 2008). Aplica para tanques "grandes horizontales o verticales soldados en el campo, aéreos que operan a presiones en el espacio vapor menores a $1,5 \mathrm{psi}$ y a temperaturas no superiores a $121^{\circ} \mathrm{C}$ " (Carreño \& Hernández, 2008, p. 49).

El material que usualmente se utiliza para la construcción de estos tanques es el acero. La selección de este dependerá del espesor a utilizar. Con respecto a la boquilla, los tanques pueden tener su boquilla inferior a nivel del fondo, abajo del tanque o en dirección a un sumidero (Carreño et al., 2008). Lo anterior, debido a que la mayoría trabaja con descarga por gravedad.

Carreño et al. (2008) mencionan que, entre los datos que se deben conocer para la realización del diseño, los principales son los correspondientes al fluido a almacenar y las condiciones de trabajo. El volumen, temperatura, peso específico del líquido y corrosión permisible son parte de la información mínima necesaria para realizar el diseño.

En el mercado se puede encontrar gran variedad de fabricantes de tanques de almacenamiento de asfalto. Se manejan varios tipos, diferentes accesorios que benefician al modificado, un rango amplio de medidas y la posibilidad de adecuarse a las necesidades del cliente. A continuación, se presentan algunos tipos de tanque y sus características dimensionales, categorizados según su fabricante (Cuadro 1).
Como mencionan Spritzer \& Guzey (2017a), los tanques tienen una caracterización la cual es referida como relación de aspecto. Para ésta se toma en cuenta la relación entre la altura y el radio. Las relaciones consideradas bajas corresponden a tanques anchos y las altas, a tanques delgados. Estos tanques se comportan de manera diferente en varios ámbitos.

Con base en lo mencionado por los mismos autores, los tanques anchos, o dicho de otra forma, tanques con radios grandes, almacenan más líquido por unidad de altura que los delgados. Por lo cual es de esperar que las fuerzas de inercia totales producidas en las paredes sean mayores a las que se tendrían en el caso de un tanque con menor radio y misma altura (Spritzer \& Guzey, 2017a).

\section{Separación de Partículas en Tanques}

Existen tanques utilizados particularmente para sedimentar. La sedimentación es un proceso natural en el que las partículas más pesadas que el medio en el que se encuentran se separan por el efecto gravitacional, o dicho de otra forma se sedimentan en el fondo (Pérez, 2005).

El mismo autor indica que para el diseño de los tanques de sedimentación, se suele considerar de suma relevancia la definición del tamaño de partícula. La partícula se representa con su longitud característica, la cual es el radio o el diámetro según la ecuación que se utilice para el cálculo de su velocidad de separación. Con esta velocidad se establece un tiempo límite de sedimentación, dato que permite realizar diseños de tanques acordes a las posibilidades físicas y económicas de los proyectos (Pérez, 2005).

Cuando la separación entre partículas ocurre por efectos de la gravedad, la misma está regida por la Ley de Stokes. Esta ley establece que el deslizamiento de las partículas es limitado por las fuerzas de fricción entre fluidos (Revelo, 2015). De acuerdo con Revelo, la ecuación que expresa la velocidad de separación es la siguiente (Ecuación 1).

$$
V_{S}=\frac{2 g}{9} \frac{r^{2}\left(\rho_{p}-\rho_{l}\right)}{\mu}
$$

Donde,

$V_{s}=$ velocidad de asentamiento $(\mathrm{cm} / \mathrm{s})$

$\mathrm{g}=$ aceleración de la gravedad $\left(\mathrm{cm} / \mathrm{s}^{2}\right)$

$\mathrm{r}=$ radio de la partícula $(\mathrm{cm})$

$\rho_{p}=$ densidad del fluido pesado $\left(\mathrm{g} / \mathrm{cm}^{3}\right)$

$\rho_{l}=$ densidad del fluido liviano $\left(\mathrm{g} / \mathrm{cm}^{3}\right)$

$\mu=$ viscosidad del medio continuo, $\mathrm{cP}$ 
Cuadro 1. Comparación de tanques de almacenamiento

\begin{tabular}{|c|c|c|}
\hline Tanque & Fabricante & Características Generales \\
\hline Tanques Estacionarios & \multirow[t]{2}{*}{ Hy-Way ${ }^{1}$} & $\begin{array}{l}\text { Capacidades desde } 10000 \text { hasta } 35000 \text { galones. } \\
\text { Longitudes desde } 21^{\prime} 0^{\prime \prime} \text { hasta } 58^{\prime} 0^{\prime \prime} \\
\text { Anchos desde } 10^{\prime} 10^{\prime \prime} \text { hasta } 11^{\prime} 4^{\prime \prime} \\
\text { Alturas de } 11^{\prime} 4^{\prime \prime} \text { hasta } 11^{\prime} 10^{\prime \prime}\end{array}$ \\
\hline Tanques Verticales & & $\begin{array}{l}\text { Capacidades desde } 10000 \text { hasta } 45000 \text { galones. } \\
\text { Anchos desde } 12^{\prime} 0^{\prime \prime} \text { hasta } 14^{\prime} 0^{\prime \prime} \\
\text { Alturas de } 14^{\prime} 9^{\prime \prime} \text { hasta } 46^{\prime} 0^{\prime \prime}\end{array}$ \\
\hline Tanques Verticales & \multirow[b]{2}{*}{ Heatec ${ }^{2}$} & $\begin{array}{l}\text { Capacidades desde } 10000 \text { hasta } 35000 \text { galones. } \\
\text { Diámetros de } 11^{\prime} 11^{\prime \prime} \\
\text { Alturas de } 16^{\prime} 11^{\prime \prime} \text { hasta } 50^{\prime} 1^{\prime \prime}\end{array}$ \\
\hline Tanques Estacionarios Horizontales & & $\begin{array}{l}\text { Capacidades desde } 10000 \text { hasta } 40000 \text { galones. } \\
\text { Longitudes desde } 18^{\prime} 4^{\prime \prime} \text { hasta } 66^{\prime} 4^{\prime \prime} \\
\text { Anchos desde } 11^{\prime} 1-1 / 2^{\prime \prime} \text { hasta } 11^{\prime} 6-1 / 2^{\prime \prime} \\
\text { Altura de } 11^{\prime} 11^{\prime \prime}\end{array}$ \\
\hline Tanques Horizontales & \multirow{2}{*}{ Highland Tank ${ }^{3}$} & $\begin{array}{l}\text { Capacidades desde } 185 \text { hasta } 70000 \text { galones. } \\
\text { Diámetros desde 3' 2" hasta 13' } \\
\text { Longitudes desde } 3^{\prime} 4^{\prime \prime} \text { hasta } 72^{\prime}\end{array}$ \\
\hline Tanques Verticales & & $\begin{array}{l}\text { Capacidades desde } 185 \text { hasta } 57500 \text { galones. } \\
\text { Diámetros desde } 3^{\prime} 2^{\prime \prime} \text { hasta 14' } \\
\text { Alturas desde } 3^{\prime} 4^{\prime \prime} \text { hasta } 50^{\prime}\end{array}$ \\
\hline
\end{tabular}

'Fuente: Gencor.com

${ }^{2}$ Fuente: Heatec.com

${ }^{3}$ Fuente: Can-am.net

Esta velocidad resulta útil para conocer acerca del posible comportamiento que presentarán los componentes del modificado ante la carencia de agitación en el tanque (caso crítico de almacenamiento). La ecuación anterior evidencia parámetros de peso para el diseño de asfaltos modificados, relacionando los asfaltos con los polímeros.

\section{Diseño del Tanque}

Inicialmente, se realiza un análisis de las relaciones altura/diámetro encontradas en las dimensiones de los tanques comerciales, mostrado en el Cuadro 2. En el caso de los tanques horizontales, se realizó la variante de sacar las relaciones con la variable longitud en el numerador y, para el diámetro, la consideración especificada en la parte inferior del cuadro. Para efectos de comparación, se considera que las dimensiones dadas por el fabricante como ancho son homólogas al diámetro en el caso de los tanques verticales. Las dimensiones de los tanques fueron convertidas a metros previamente. 
Cuadro 2. Relaciones dimensionales en tanques comerciales

\begin{tabular}{|c|c|c|c|}
\hline & Fabricante & Altura/Diámetro & Dimensiones (m) \\
\hline \multirow{3}{*}{ Tanques verticales } & Hy-Way & $1,23-3,28$ & $\begin{array}{l}\text { Altura: 4,496 m hasta } 14,021 \mathrm{~m} \\
\text { Diámetro: - } \\
\text { Ancho: } 3,658 \mathrm{~m} \text { hasta } 4,267 \mathrm{~m}\end{array}$ \\
\hline & Heatec & $1,42-4,20$ & $\begin{array}{l}\text { Altura: 5,156 m hasta } 15,265 \mathrm{~m} \\
\text { Diámetro: } 3,632 \mathrm{~m} \\
\text { Ancho: - }\end{array}$ \\
\hline & Highland Tank & $1,05-3,57$ & $\begin{array}{l}\text { Altura: 1,016 m hasta } 15,24 \mathrm{~m} \\
\text { Diámetro: 0,965 m hasta 4,267 m } \\
\text { Ancho: - }\end{array}$ \\
\hline \multirow{3}{*}{ Tanques Horizontales } & Hy-Way & $1,89-5,01$ * & $\begin{array}{c}\text { Altura: 3,454 m hasta } 3,607 \text { m } \\
\text { Longitud: 6,401 m hasta 17,678 m } \\
\text { Diámetro: - } \\
\text { Ancho: } 3,302 \text { m hasta } 3,454 \mathrm{~m}\end{array}$ \\
\hline & Heatec & $1,60-5,68$ * & $\begin{array}{c}\text { Altura: } 3,632 \mathrm{~m} \\
\text { Diámetro: }- \\
\text { Longitud: } 5,588 \mathrm{~m} \text { hasta } 20,218 \mathrm{~m} \\
\text { Ancho: } 3,366 \mathrm{~m} \text { hasta } 3,492 \mathrm{~m}\end{array}$ \\
\hline & Highland Tank & $1,05-5,54$ & $\begin{array}{l}\text { Altura: - } \\
\text { Diámetro: } 0,965 \mathrm{~m} \text { hasta 3,962 m } \\
\text { Longitud: } 1,016 \mathrm{~m} \text { hasta } 21,946 \\
\text { Ancho: - }\end{array}$ \\
\hline
\end{tabular}

*Para esta relación en tanques de diseño elíptico, se sacó un promedio entre el ancho y la altura de la base mínimos y máximos, para considerarlos como un rango de diámetros promedio.

(Hy-Way) Diámetro: 3,378 m hasta 3,530 m

(Heatec) Diámetro: 3,499 m hasta 3,562 m

Tal como es posible observar en el Cuadro 2, las relaciones dimensionales para el caso de los tanques de almacenamiento verticales oscilan entre 1,05 y 4,20. Paralelamente, las relaciones para tanques horizontales se encuentran en un rango entre 1,05 hasta 5,68. Ambas relaciones resultaron con un mínimo igual y con valores máximos menores a 6,0. Para considerar la relación de aspecto de estos tanques comerciales tal y como lo señala la teoría de Spritzer y Guzey (2017a) se muestra el Cuadro 3.

Cuadro 3. Relaciones de aspecto de los tanques comerciales encontrados

\begin{tabular}{|c|c|c|}
\hline \multicolumn{2}{|c|}{ Relaciones de Aspecto } \\
\hline \multirow{2}{*}{ Fabricante } & Verticales & Horizontales \\
\cline { 2 - 3 } & Altura/Radio & $3,78-10,02$ \\
\hline Hy-Way & $2,46-6,56$ & $3,20-11,36$ \\
\hline Heatec & $2,84-8,40$ & $2,10-11,08$ \\
\hline Highland Tank & $2,10-7,14$ & Longitud/Radio \\
\hline
\end{tabular}


En otro artículo de Spritzer y Guzey (2017b), los autores señalan una distinción de 0,4 (anchos) y 2,0 (delgados). A partir de esto se puede decir que los tanques comerciales consultados son todos considerados delgados.

Se destaca el hecho de que se buscan condiciones críticas para obtener resultados para los peores escenarios, en este caso omitiendo la agitación en el producto durante su almacenamiento. El comportamiento del modificado en un tanque sin agitación puede presentar separación de fases, lo cual se puede relacionar con el comportamiento de una mezcla en almacenamiento que experimenta sedimentación. Ante este fenómeno es necesario mencionar que la acumulación de partículas separadas en ciertas secciones del fluido aumenta la concentración de las mismas, lo que resultará en una disminución de su velocidad de separación, razón por la que no puede estudiarse como una simple caída de sólidos en un líquido (Martín, Salcedo \& Font, 2011). En la Figura 3 se muestra la sedimentación discontinua mediante un experimento en una probeta de vidrio.

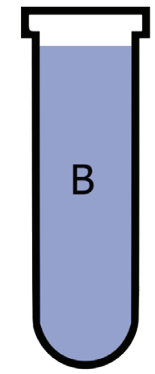

(a)

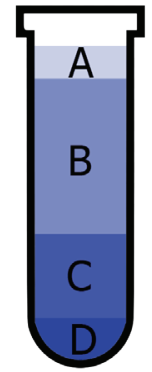

(b)

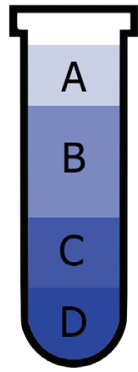

(c)

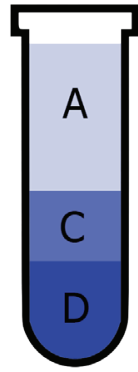

(d)

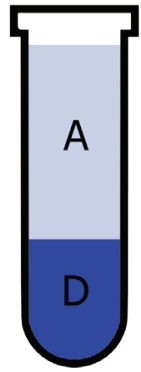

(e)
Figura 3. Fases de un experimento de sedimentación discontinua, desde los 0 segundos hasta su completa sedimentación. Se describen las secciones: B, concentración inicial; A, libre de sólidos; C, sólidos sedimentan sin contacto continuo; D, lodo concentrado (Basada en Martín et al., 2011)
La Figura 3.a representa el momento inicial del proceso, en la secuencia de imágenes se observan las fases con distintas concentraciones de la suspensión. Elcolor más oscuro representa la zona con más concentración. Conforme transcurre el tiempo el espesor de las capas varía (Martín et al., 2011).

Con el fin de propiciar la concentración crítica para obtener espesores de capa más distinguibles entre sí, se considera más adecuado diseñar un tanque vertical delgado (alto y de diámetro pequeño). Al tener una concentración crítica, se entra en el rango de sedimentación impedida, rango en el cual las mismas partículas de la suspensión interactúan unas con otras, lo que disminuye la velocidad en que sedimentan (Martín et al., 2011). Dicho en otras palabras, las mismas partículas ralentizan su propia sedimentación. Por lo que un área de base pequeña evidenciará mejor las diferentes concentraciones mediante espesores de capas más gruesos que los que puedan ocurrir en tanques anchos u horizontales.

Ahora, para la construcción del tanque de almacenamiento para estudio, considerando diámetros de tubos comerciales de acero, se seleccionó un diámetro de base de 3 pulgadas (7,62 $\mathrm{cm}) \mathrm{y}$, acorde a la cantidad de asfalto modificado con el que se trabajará, una altura de $22 \mathrm{~cm}$ de volumen contenido. En lo que respecta a la altura real del tanque, la misma será de $25 \mathrm{~cm}$, considerando un área libre y espacio para colocar una tapa. En conjunto con los soportes la altura total del tanque de almacenamiento diseñado es de 35,15 cm.

La cantidad total de asfalto con la cual se contará es de 1 galón (3,78541 L). Este se repartirá hasta tener tres tanques de almacenamiento a escala con un litro de asfalto modificado cada uno.

Evaluando las relaciones del tanque diseñado para trabajar a nivel de laboratorio se obtiene la información detallada en el Cuadro 4.

Cuadro 4. Relaciones de tanque diseñado para trabajo en laboratorio

\begin{tabular}{|c|c|c|}
\hline Dimensiones & Altura/Radio & Altura/Diámetro \\
\hline Diámetro: $0,0762 \mathrm{~m}$ & & \\
Altura: $0,25 \mathrm{~m}$ & 6,56 & 3,28 \\
Capacidad: $0,001 \mathrm{~m}^{3}$ & & \\
\hline
\end{tabular}


Con las relaciones obtenidas en el Cuadro 4 se observa que la relación de altura entre el diámetro está dentro del rango que cumplen los tanques comerciales verticales, es decir entre 1,05 a 4,20. La relación de aspecto es mayor a 2, por lo que se trata de un tanque delgado, acorde a lo buscado para propiciar una buena distinción entre las capas al separarse.

Para la toma de muestras en el diseño se instalan tres válvulas. Estas son colocadas a los tres niveles de interés para el presente estudio, los cuales son: al tope del volumen contenido, a la mitad y una inferior de la cual se tomarán las muestras del fondo. Se eligen válvulas de $1 / 4$ " tipo llave italiana, por su diámetro pequeño, cuyo volumen a obtener alcanza para las muestras a estudiar y por su simpleza de uso al momento de recolectar los especímenes.

Se trabaja con acero inoxidable debido a su resistencia al calor y a la corrosión, así como su facilidad de limpieza. La utilización de un tubo y válvulas de medidas comerciales se debe a su fácil accesibilidad.

Debido al diseño, cabe mencionar que una base estable que dé un buen soporte es necesaria para poder mantener estos tanques adecuadamente en el horno y tomar las muestras sin inconvenientes. Esta base fue diseñada de planchuelas de hierro soldadas a un cinturón metálico de refuerzo rodeando al tubo.

El diseño propuesto del tanque de almacenamiento de asfalto modificado a escala se detalla en los planos adjuntos como anexos. Allí, se muestran las cotas dimensionales y ciertas especificaciones, con el propósito de detallar ciertos aspectos relevantes de diseño. La vista del tanque que se propone en este documento se presenta en la Figura 4.

Una particularidad del tanque se encuentra en la toma de muestras inferior. Con el fin de sacar realmente lo que se encuentra en el fondo, la misma se ubicó saliendo de un agujero en el medio del fondo, direccionando el fluido por un conducto que se ajusta a un ángulo de $45^{\circ}$, tal como se puede observar en la Figura 5.

El principal interés radica en contrastar las diferencias que van a ir ocurriendo entre el asfalto modificado encontrado en la parte más alta del tanque y la más baja, considerando también el comportamiento al medio. Para las muestras a nivel superior, se utilizará una salida (válvula) colocada para que coincida lo mejor posible con lo que será el tope del volumen contenido, en este caso coincidiendo la parte superior del agujero de la válvula con los 22 centímetros que respectan al tope del volumen de muestra. La salida del medio tendrá su centro concéntrico a la mitad, lo que resulta en 11 centímetros de la altura del volumen de asfalto modificado contenido. Lo anterior se puede evidenciar más fácilmente en la Figura 6.

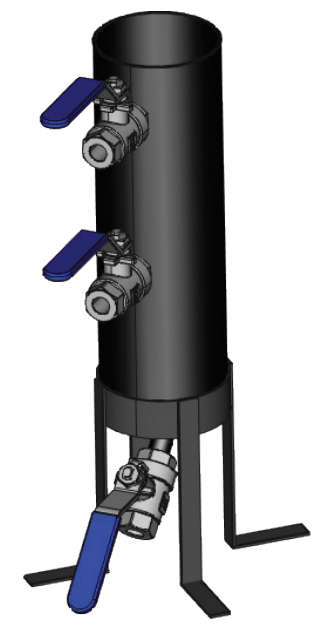

Figura 4. Tanque de almacenamiento de asfalto a escala para estudio de separación

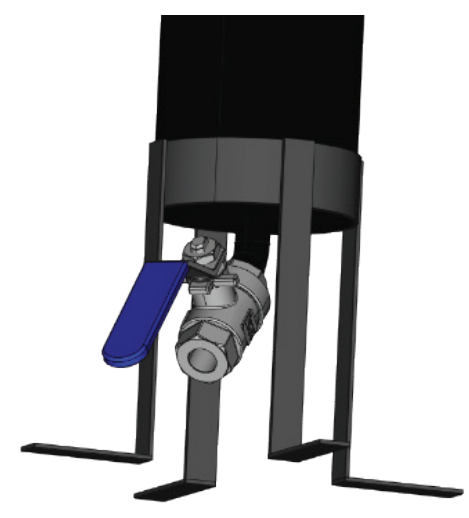

Figura 5. Diseño de toma de muestras inferior del tanque de almacenamiento de asfalto

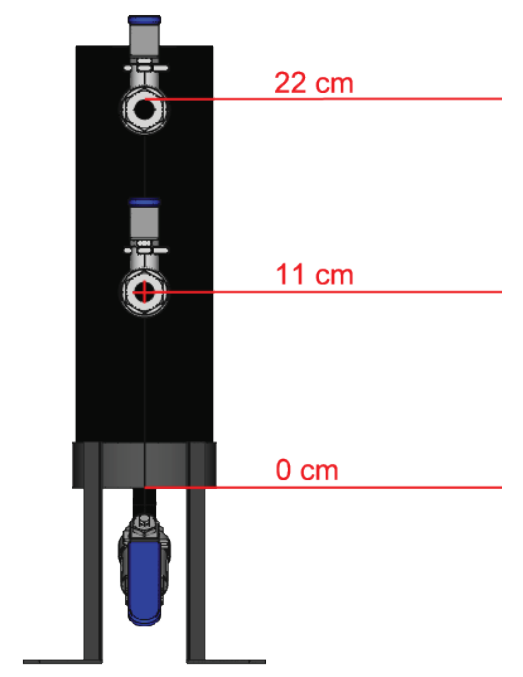

Figura 6. Ubicación de los agujeros superior y medio en el tanque 
Propuesta para el estudio de separación de fases del asfalto modificado

Con respecto al análisis de datos, utilizando la ecuación 1, considerando las propiedades del asfalto a evaluar y las del polímero con el cual se modificará y de acuerdo con las temperaturas de almacenamiento, se puede calcular una velocidad a la cual las partículas se separarán en condiciones de reposo. Se debe tomar en cuenta, además, la profundidad que el asfalto modificado tendrá en el tanque diseñado. Así, se tendrá una idea de la composición que se espera encontrar después de los diferentes intervalos de tiempo.

Es recomendado que, según este análisis, el lector establezca los tiempos de toma de muestra y estudio del asfalto modificado, para que se adecúe más a las condiciones que su caso presente. Otra consideración referente a los tiempos a los cuales realizar las pruebas propuestas en la presente investigación, es la que aportan los tiempos utilizados en el artículo de Villegas-Villegas et al. (LanammeUCR, 2013). En este documento el ensayo se realizó considerando desde las 2 horas hasta las 196 que transcurrieron. También se considera pertinente rescatar que en esa investigación se obtuvo que aproximadamente después de las 24 horas ya todo el polímero se encontraba separado del asfalto.

Este estudio propone que se realice la comparación de muestras recolectadas en diferentes lapsos de tiempo por cada tanque para el análisis de la cinética del asfalto modificado. Para esto, se debe tomar la muestra inicial del asfalto recién modificado. Luego, distribuir la mezcla en los tres tanques de manera equitativa, identificarlos bien y colocarlos en el horno. Según los lapsos definidos para toma de muestras, tomar una muestra por nivel del tanque que corresponda (tres muestras en total) durante el tiempo transcurrido. Es importante que dicha acción se realice en el siguiente orden: muestra superior, muestra del medio y muestra inferior. En este orden se alterará mínimamente la mezcla. Al concluir, realizar un estudio de las viscosidades e identificar las diferencias entre las nueve muestras obtenidas, considerando sus concentraciones. Se deben analizar después por nivel y según el tiempo transcurrido desde el inicio de su almacenamiento.

El principal propósito del presente estudio radica en estudiar el comportamiento de asfaltos modificados durante su almacenamiento ya que, cuando este producto no tiene las características adecuadas, se repercute enormemente en los proyectos para los cuales es seleccionado y, durante el periodo de almacenamiento se corre el riesgo de que pierda sus propiedades. Los autores del presente documento se han propuesto el objetivo de crear un modelo que brinde información con respecto a la calidad y la vida útil del modificado, determinando tiempos en los cuales, ante escenarios críticos, su composición no se vea comprometida con respecto a la del diseño original. Dicho proyecto se está trabajando en busca de aportar a los diseños de laboratorio y al control de calidad del proceso en planta, mediante la utilización de este diseño y de la propuesta de análisis de las muestras dada.

\section{CONCLUSIONES}

Como parte del objetivo principal del documento, se propone el estudio de la cinética del asfalto modificado, describiendo los pasos a seguir para la toma de muestras y los análisis a realizar. Siguiendo esta propuesta, se tienen los primeros pasos para el planteamiento de un modelo que pretende identificar el comportamiento que presenta un asfalto modificado durante su almacenamiento, con el fin de definir tiempos máximos de almacenaje del producto en los cuales sus propiedades de diseño no se vean comprometidas.

Siendo este estudio a condiciones críticas, se espera el peor de los escenarios, por lo que se recomienda que la última toma de muestras no se realice más allá del día en almacenamiento transcurrido, ya que no aportaría mucho al estudio. También se recuerda al lector que la ecuación que determina la velocidad de separación de partícula, presentada en este documento, puede ser utilizada para la selección de los tiempos de toma de muestras a definir para este ensayo.

Para propiciar la separación de fases y facilitar el estudio del fenómeno, se decidió diseñar un tanque delgado, es decir alto y de base pequeña. Sus relaciones dimensionales de altura/ diámetro y altura/radio fueron 3,28 y 6,56 , respectivamente. Estas relaciones están dentro del rango presente en los tanques comerciales y lo categorizan como un tanque delgado.

El tanque diseñado considera tres alturas diferentes para la toma de muestras con la intención de alterar mínimamente el volumen almacenado. Se construye con componentes de fácil obtención y dimensiones normalizadas, buscando asegurar su optimo desempeño, así como simpleza al utilizar. Entre su diseño destaca la válvula inferior, la cual permite facilitar la salida del polímero por la misma en caso de que este sedimentara, asegurando una concentración real de la muestra y, por tanto, sus propiedades viscoelásticas. 
Entre las recomendaciones para investigaciones relacionadas se tiene que, aunque la selección del tanque vertical para el estudio del caso crítico se debe a que su diseño acelera los tiempos de separación de las fases en el asfalto modificado, un estudio del comportamiento de este material en un tanque horizontal puede ser valioso. Siendo que, a nivel industrial, los tanques verticales son más usados para almacenar grandes cantidades del material, se destaca también que la representación y el estudio en un tanque no vertical va ligado más a la realidad del cómo se transporta y se almacena en pequeñas plantas, por lo que se podría estudiar el efecto de su tiempo de almacenado y traslado en el mismo, así como también evaluar la incidencia que puede tener en la separación de fases.

De ser posible, se podría también considerar el uso de software de simulación para el estudio del comportamiento del asfalto modificado en las condiciones a las cuales viaja el fluido en el tanque horizontal para su posterior utilización.

\section{REFERENCIAS}

Angulo, R. y Duarte, J. (2005). Modificación de un asfalto con caucho reciclado de llanta para su aplicación en pavimentos. (Tesis de grado), Universidad Industrial de Santander, Bucaramanga.

Carreño, G. y Hernández, J. (2008). Diseño y Calculo de un Tanque de Almacenamiento para Nafta con Diámetro de $70 f t$ x 30ft de Altura Bajo la Norma API 650. (Tesis de grado). Instituto Politécnico Nacional, México.

Fu, H., Xie, L., Dou, D., Li, L., Yu, M., y Yao, S. (2006). Storage stability and compatibility of asphalt binder modified by SBS graft copolymer. Construction and Building Materials, 21(7), 1528-1533. doi:10.1016/j.conbuildmat.2006.03.008

Heatec. (s.f.). Asphalt Storage Tanks. Chattanooga, EU.:Astec Industries Company. Recuperado el 09 de junio de 2020, de: http:// heatec.com/literature/brochures/HeatecAsphaltTanks.pdf

Highland Tank. (s.f.). Petroleum E Chemical Storage Tanks. Recuperado el 09 de junio de 2020, de: https://can-am.net/wp-content/ uploads/petroleum_chemical_storage_tanks.pdf

Hy-Way. (s.f.). Liquid Storage Tanks. Florida, EU.: GENCOR. Recuperado el 09 de junio de 2020, de: https://www.gencor.com/wpcontent/uploads/2019/04/Liquid-Storage-Tank-REV18.pdf

Martín, I., Salcedo, R., y Font, R. (2011). Mecánica de Fluidos Tema 5. Operaciones separación sólido-fluido. Universidad de Alicante.

Pérez, L. (2005). Teoría de la sedimentación. Instituto de Ingeniería Sanitaria y Ambiental. Recuperado de: http://www.fi.uba.ar/ archivos/institutos_teoria_sedimentacion.pdf

Rassamdana, H., Dabir, B., Nematy, M., Farhani, M., y Sahimi, M. (1996). Asphalt flocculation and deposition: I. The onset of precipitation. AIChE Journal, 42(1), 10-22. doi:10.1002/aic.690420104

Revelo, I. (2015). Diseño de tanques, usados en la separación del agua de formación para el proceso de reinyección, estación Secoya. (Tesis de grado). Universidad Central del Ecuador, Quito.

Spritzer, J., y Guzey, S. (2017a). Review of API 650 Annex E: Design of large steel welded aboveground storage tanks excited by seismic loads. Thin-Walled Structures, 112, 41-65. doi:10.1016/j.tws.2016.11.013

Spritzer, J. M., y Guzey, S. (2017b). Nonlinear numerical evaluation of large open-top aboveground steel welded liquid storage tanks excited by seismic loads. Thin-Walled Structures, 119, 662-676. doi:10.1016/j.tws.2017.07.017

LanammeUCR. (2013). Estabilidad al almacenamiento de asfaltos modificados en función del cambio en sus propiedades reológicas y su distribución de partículas según la microscopia de fuerza atómica. Recuperado de: https://www.lanamme.ucr.ac.cr/ repositorio/handle/50625112500/537

Federal Highway Administration. (2005). Evaluation of the laboratory asphalt stability test (No. FHWA-HRT-04-111) 
ANEXO. Tanque de almacenamiento de asfalto laboratorio.

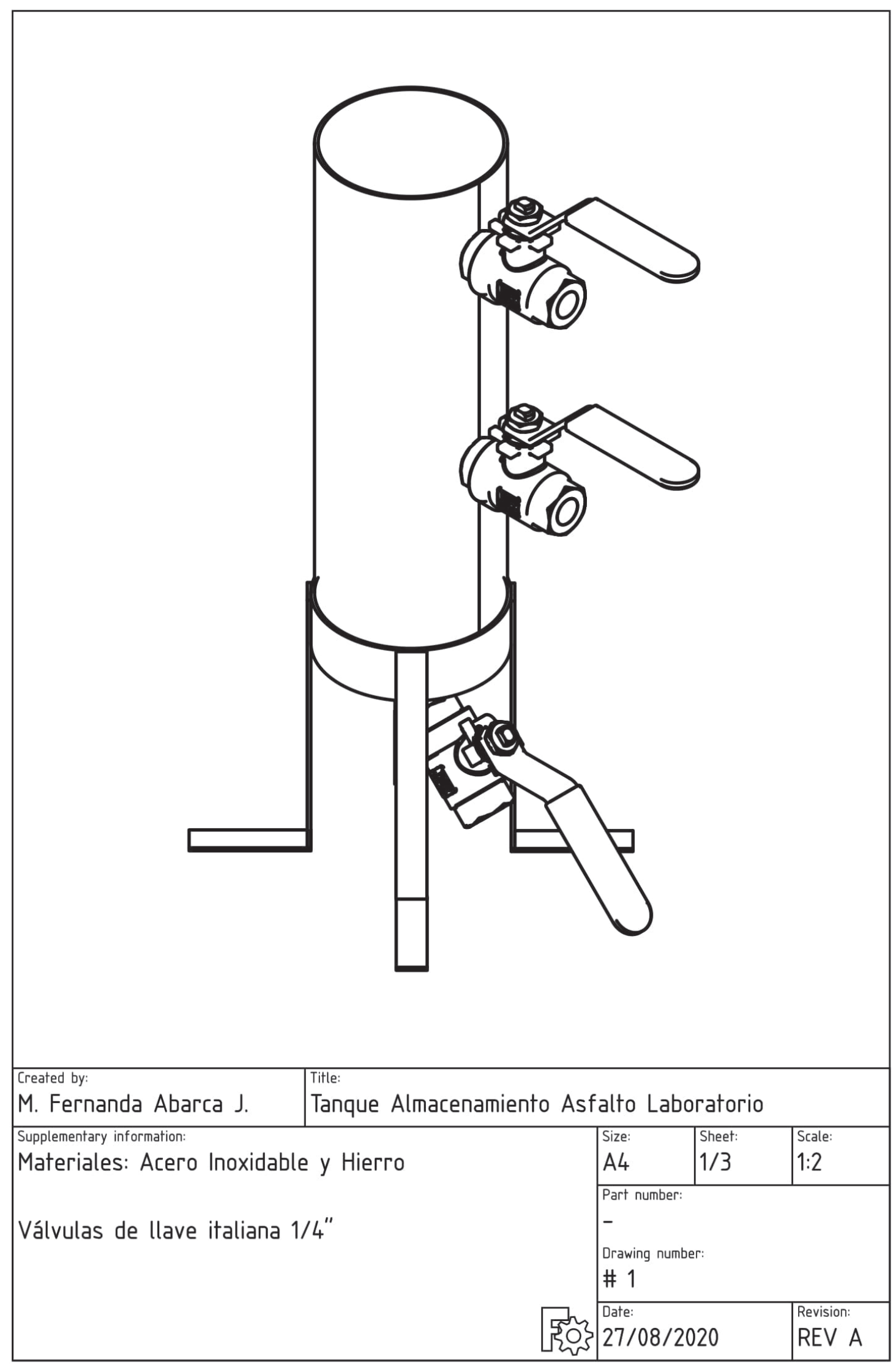

10

Revista Infraestructura Vial / LanammeUCR / ISSN electrónico: 2215-3705 / Volumen 23 / Número 42 / Julio, 2021 


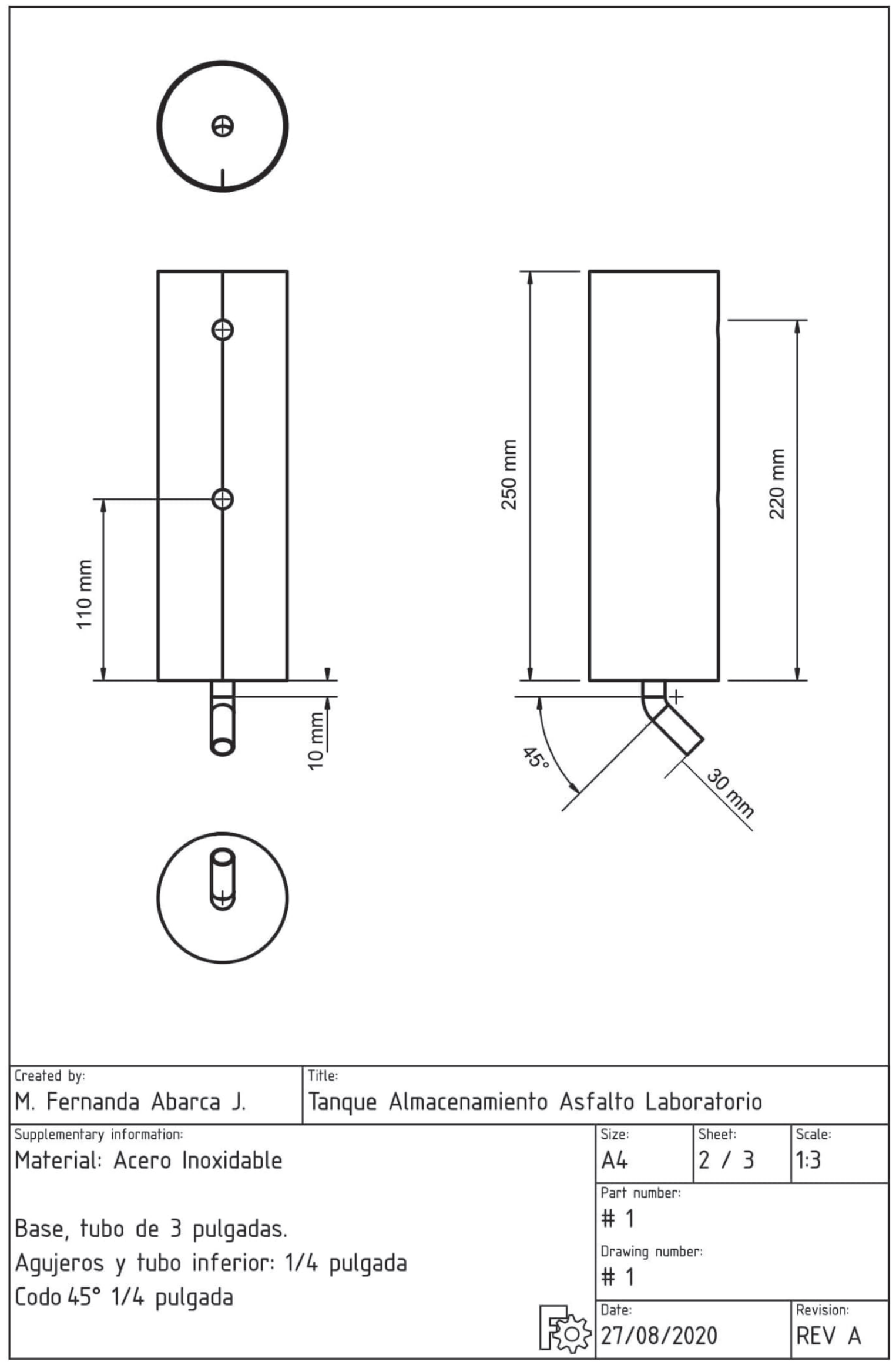




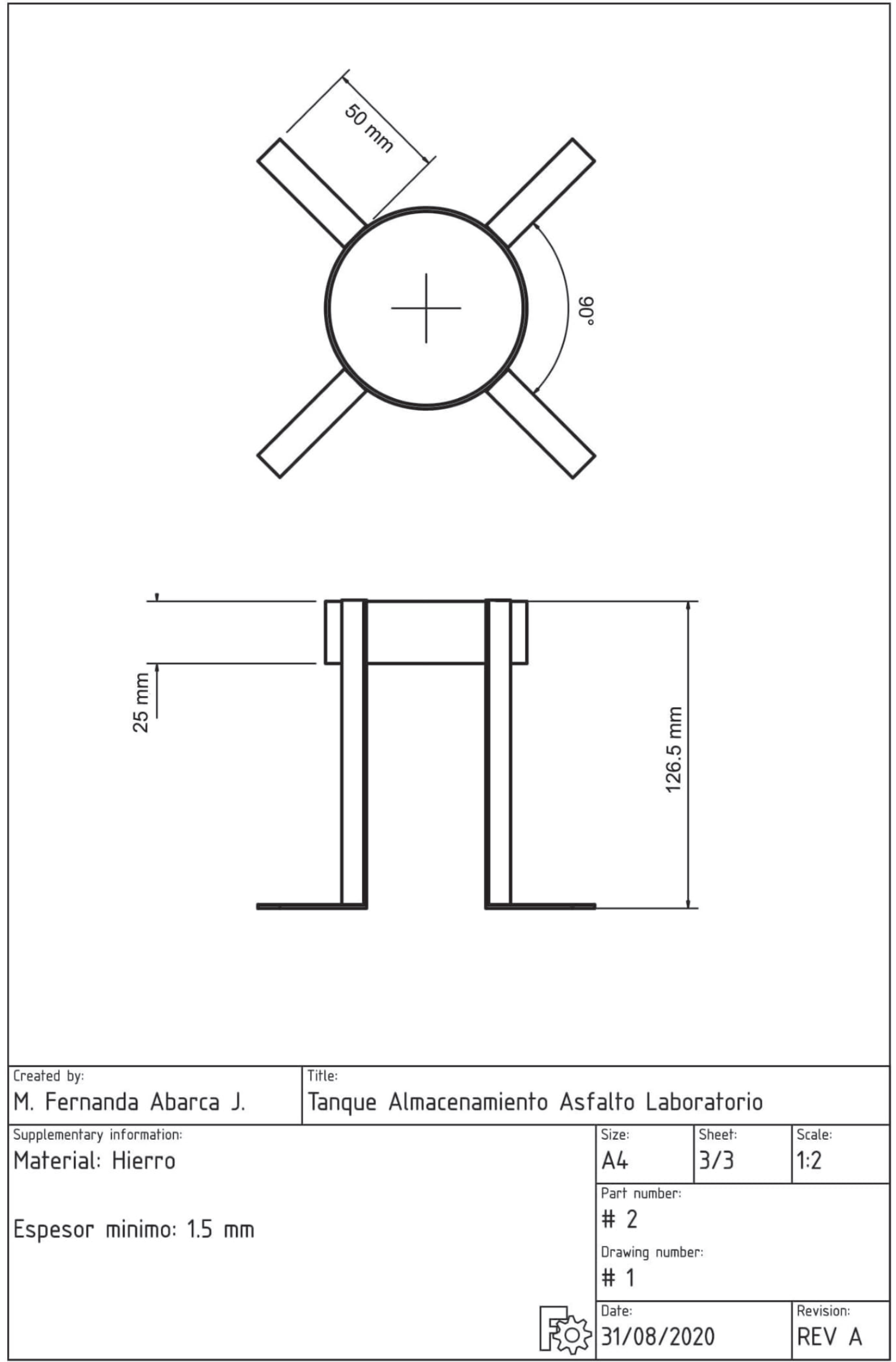

